\section{Uveoscleral outflow}

The balance between inflow and outflow of aqueous humour determines the level of the intraocular pressure (IOP). There is a single source of inflow: the secretion of aqueous flow from the ciliary processes. However, for more than 30 years it has been known that there are two alternative routes for outflow: the conventional outflow route through the trabecular meshwork and the unconventional or uveoscleral outflow route. The latter was first described by Bill, ${ }^{1,2}$ who reported that in the cynomolgus monkey about half the aqueous did not leave the anterior chamber through the trabecular meshwork but left the eye by passing through the ciliary muscle into the supraciliary and suprachoroidal spaces. Although Bill and Phillips ${ }^{3}$ demonstrated uveoscleral flow in the human eye also, it was generally not considered of clinical importance. There were probably several reasons for the lack of interest in uveoscleral flow among clinicians. The report indicated that in the human eye only a small proportion of the aqueous left the eye through this route. Another reason might be that there was no non-invasive technique that could be used clinically to study uveoscleral flow in the human eye. Now the introduction of prostaglandin analogues in the treatment of glaucoma has renewed the interest in this outflow route, and a reduction in the resistance to flow through the ciliary muscle is accepted as an efficient way to reduce the IOP.

\section{Techniques to measure uveoscleral flow}

Aqueous leaves the eye by bulk flow and large molecules are not selectively retained. Small molecules, on the other hand, can be lost by diffusion into surrounding tissues. However, for the normal constituents of the aqueous there is no net loss. Diffusion will occur in both directions and what is lost into surrounding tissues will be compensated for by diffusion into aqueous from those tissues. Still, for a small tracer introduced into the aqueous a certain loss

Albert Alm, MD, PhD Department of Neuroscience, Ophthalmology University Hospital

S-701 85 Uppsala

Sweden

e-mail:

Albert.alm@ogon.uas.lul.se by diffusion must be expected. Thus about $10 \%$ of sodium fluorescein introduced into the anterior chamber is lost by diffusion and calculations of aqueous flow based on fluorophotometry must take that into account. ${ }^{4}$ A suitable tracer for measuring aqueous flow in experimental animals should therefore be large enough to avoid loss by diffusion.

\section{ALBERT ALM}

In monkeys radioactively labelled albumin injected or infused into the anterior chamber is commonly used. The route by which the albumin leaves the eye can be determined by following its appearance in plasma. The albumin that leaves the eye through the trabecular meshwork will appear immediately in plasma. The albumin that leaves the eye through the uveoscleral route will be retained within the supraciliary and suprachoroidal spaces for several hours. The amount that leaves the eye through the two outflow routes can then easily be determined by following the amount of tracer appearing in plasma and determining the amount of radioactivity left in the anterior uvea after a few hours. If the amount of tracer introduced into the anterior chamber is known, the amount leaving through the uveoscleral route can be calculated by subtracting the amount appearing in plasma from the total amount of tracer injected into the anterior chamber. ${ }^{5}$

Obviously this technique can not be used clinically, although some human eyes scheduled for enucleation have been used to verify that uveoscleral flow takes place also in human eyes. ${ }^{3}$ Nevertheless there are a number of clinical techniques for determining the various components of aqueous humour dynamics. The Goldmann equation, adjusted for uveoscleral flow, establishes the relationship between IOP, aqueous flow $\left(F_{\mathrm{IN}}\right)$, uveoscleral flow $\left(F_{U S}\right)$, outflow facility through the trabecular meshwork $(C)$, and episcleral venous pressure $\left(P_{\mathrm{EV}}\right)$ :

$$
\mathrm{IOP}=P_{\mathrm{EV}}+\left(F_{\mathrm{IN}}-F_{\mathrm{US}}\right) / C
$$

All but $F_{\text {US }}$ can be determined clinically. Theoretically one could then measure the other parameters and calculate $F_{\text {Us. }}$ In reality this is not feasible since the measurement errors involved are too great to provide meaningful

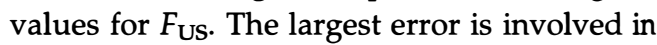
measuring $C$. Toris et al..$^{6}$ have introduced a technique whereby $C$ is calculated by measuring IOP and $F_{\mathrm{IN}}$ at two different levels of IOP and $F_{\mathrm{IN}}$. They measured IOP and $F$ before and after a combination of a topical nonselective beta-blocker and a full dose of acetazolamide. Neither drug is expected to change $F_{\mathrm{US}}, C$ or $P_{\mathrm{EV}}$. Assuming a value for $P_{\mathrm{EV}}$ of $9 \mathrm{mmHg}, C$ can then be calculated as $\Delta \mathrm{F}_{\mathrm{IN}} / \Delta \mathrm{IOP}$. Thus this technique is independent of clinical tonography, a technique that is 
influenced not only by changes in outflow resistance through the trabecular meshwork but also by changes in resistance through the uveoscleral route and by pseudofacility. The technique should be particularly useful in eyes where the IOP is much larger than $P_{\mathrm{EV}}$. When IOP and $P_{\mathrm{EV}}$ are similar, the variability of the technique is still substantial.

\section{Normal values}

The amount of aqueous leaving through the uveoscleral route seems to be related to the development of the ciliary muscle. Consequently its much higher in monkeys, at about $50 \%$ of total outflow, ${ }^{1,2}$ than in rabbits, where it is only about $5 \%$ of total outflow. ${ }^{7}$ In the study with labelled albumin in human eyes uveoscleral flow was determined in only two eyes without any pharmacological intervention and in those two eyes $4 \%$ and $14 \%$ of the flow left the eye through the uveoscleral route. ${ }^{3}$ Thus from these data uveoscleral flow seems to be less prominent in human eyes than in monkey eyes. However, that may be an age-related difference rather than a species difference. Experimental animals are usually young and Toris and co-workers ${ }^{8}$ have reported lower values calculated from fluorophotometric measurements, with $1.52 \mu \mathrm{l} / \mathrm{min}$ in normal eyes in the age group $20-30$ years and only $1.10 \mu \mathrm{l} / \mathrm{min}$ in normal individuals 60 years old or older.

\section{Physiology of uveoscleral flow}

The choroidal vessels are unusually permeable and permit passage even of large proteins. The protein concentration in the extravascular space of the choroid is also high, being about $60 \%$ of that in plasma. ${ }^{9}$ This results in a high colloid osmotic pressure in the extravascular fluid of the choroid and a pressure gradient across the retinal pigment epithelium favouring absorption of fluid from the retina. This is likely to be a contributory factor to the adherence of the retina to the pigment epithelium and in the prevention of retinal oedema. As there is a constant, although slow, delivery of proteins into the extravascular space of the choroid there has to be a removal and there is a slow turnover of proteins in the extravascular spaces of the uvea. Lymph vessels normally remove extravascular proteins. No such vessels exist in the eye but the permeable sclera and the pressure gradient caused by the difference in IOP and pressure in the orbit permit loss of even large proteins through the sclera. In fact, the routes through the ciliary muscles are also large enough to permit passage of small spheres ${ }^{10}$ and aqueous leaves the eye through the uveoscleral route by bulk flow, as is the case for the trabecular meshwork. Thus the composition of the aqueous is not changed during its passage through the outflow routes, but small molecules are exchanged by diffusion with surrounding tissues.

Bulk flow is always pressure dependent. It is nevertheless often said that uveoscleral flow is not pressure dependent. In terms of the pressure gradient between the IOP and the orbit this is true as long as the IOP is at least $4 \mathrm{mmHg}$. ${ }^{11}$ Thus the uveoscleral flow rate is about the same at normal and high IOP. For flow through the trabecular meshwork this is not the case, as the higher the IOP the larger the flow through the trabecular meshwork. The explanation is best understood if the uveoscleral flow route is considered as two routes, coupled in series. The first is from the anterior chamber to the suprachoroidal space, the second from the suprachoroidal space into the orbit. As IOP increases the pressure in the suprachoroidal space will increase to the same extent, which means that the pressure gradient for the first part of the uveoscleral flow will not increase and consequently flow into the suprachoroidal space will not increase. Flow through the sclera will increase, but the fact that total uveoscleral flow does not increase with higher IOP indicates that the first step - into the suprachoroidal space - is the ratelimiting one. Also, aqueous that reaches the suprachoroidal space may be absorbed by the uveal vessels. The amount of aqueous leaving the eye through the ciliary muscle - in the order of $1 \mu \mathrm{l} / \mathrm{min}$ - is very small compared with uveal blood flow.

The purpose of the uveoscleral outflow route is not clear. One might question whether it is the result of a specific design or just a consequence of lack of a barrier to flow in front of the ciliary muscle. It would, in fact, require a special design to prevent it. It is useful, however, when there is inflammation in the anterior segment of the eye. Inflammation tends to produce debris that might clog the trabecular meshwork and reduce outflow through this route. At the same time the prostaglandins produced by the inflammation will reduce resistance to flow through the uveoscleral outflow route and thus increase this flow as a compensation for the reduced flow through the trabecular meshwork.

\section{Pharmacology of uveoscleral flow}

\section{The uveoscleral route and drug delivery}

The existence of a uveoscleral route provides an opening for even large molecules to reach the suprachoroidal space and there are no barriers preventing such molecules from reaching the posterior pole of the eye. In fact, when uveoscleral flow is determined with radioactively labelled albumin it is not unusual to find albumin all the way to the optic nerve. Thus one might speculate whether this route could be used to deliver drugs to the posterior pole of the eye. However, there are several factors that will make this difficult. Small molecules in the extravascular space of the choroid are rapidly washed out. The half-life in the extravascular space for a small molecule injected into the arterial side of the choroid is only a few seconds. ${ }^{12}$ One might conclude that any molecule small enough to pass through the cornea would be washed out by the large uveal blood flow before entering far into the suprachoroidal space. The situation may be different for small molecules that are bound to melanin. They will accumulate in the uvea, most likely reaching it by the 
systemic circulation, but then they will be inactivated through binding to melanin. A further problem that must be circumvented is that the retinal pigment epithelium is a barrier for most non-lipid-soluble substances. Drugs that reach the extravascular space of the choroid might reach the optic nerve head, where there is no barrier, but they will not easily pass the retinal pigment epithelium. Thus with present techniques the uveoscleral route is unlikely to present an effective route for drug delivery to the posterior pole.

\section{Cholinergic drugs}

As aqueous passing through the uveoscleral route has to pass through the ciliary muscle it is not surprising that the state of contraction of the ciliary muscle will influence the flow through it. Thus pilocarpine reduces and atropine increases uveoscleral flow in monkeys. ${ }^{13,14}$ The same is true for human eyes. Bill and Phillips ${ }^{3}$ determined $F_{U S}$ in human eyes and found that in eyes pre-treated with atropine $4-27 \%$ of the aqueous left through the uveoscleral route compared with $0-3 \%$ in eyes pre-treated with pilocarpine, and $4 \%$ and $14 \%$ respectively in two eyes with no pre-treatment.

\section{Adrenaline}

Adrenaline is a drug that seems to have a role in many aspects of aqueous humour dynamics. Adrenergic receptors, both adrenergic alpha ${ }_{2}$-receptors and beta ${ }_{2}$ receptors, are involved in regulation of aqueous flow. Adrenaline acts through the beta 2 -receptor, but it has its main effect at night when adrenergic tone is low and adrenaline will increase aqueous flow. ${ }^{15}$ However, the main mechanism of action is on outflow, which is increased, and the overall effect is a reduction of the IOP. In monkeys one early effect of adrenaline is to increase uveoscleral flow, which was doubled. ${ }^{16}$ Camras et al. ${ }^{17}$ examined the possibility that the effect on uveoscleral flow was mediated by prostaglandins and examined a group of glaucoma patients who received indomethacin or placebo apart from adrenaline. They found that the IOP reduction was significantly smaller in the group that received indomethacin and concluded that part of the effect on IOP by adrenaline was mediated by prostaglandins. However, it must be assumed that adrenaline has another form of action too since the maximal effect on IOP of a single dose of adrenaline is seen within 1-2 h, a time by which prostaglandins have very little effect on IOP.

\section{Prostaglandins}

Prostaglandin analogues have recently been introduced in the treatment of glaucoma. They are effective ocular hypotensive drugs without reducing aqueous flow. The IOP reduction is entirely due to increased outflow. Determination of outflow through the two outflow routes in monkeys has shown that both prostaglandins $F_{2 \alpha}$ and its analogue latanoprost reduce the IOP solely by increasing uveoscleral flow. ${ }^{18,19}$ There was no effect on trabecular outflow. Calculations of uveoscleral flow in human eyes also indicate that the main effect of latanoprost is on the uveoscleral outflow. ${ }^{6}$

The mechanism by which prostaglandins increase uveoscleral outflow has been investigated in cell cultures and in monkeys. Prostaglandin $\mathrm{F}_{2 \alpha}$ relaxes the ciliary muscle ${ }^{20}$ but it is unlikely that this explains much of the effect. The onset of action of prostaglandin $\mathrm{F}_{2 \alpha}$ and of latanoprost is much slower than one would expect from a direct relaxation of the ciliary muscle. Studies in vitro on ciliary muscle cell cultures and in vivo on monkey eyes have shown that prostaglandin $\mathrm{F}_{2 \alpha}$ and latanoprost induce increased secretion of prometalloproteinases (MMPs) and thus cause a rearrangement of the extracellular matrix of the ciliary muscle with a reduction of the amount of collagens and its other constituents. ${ }^{21-23}$ The effects in cell cultures are quite impressive, with up to $50 \%$ loss of collagens. In vivo they are much more modest. These effects are likely to reduce resistance of flow through the trabecular meshwork and are at the moment the most likely explanation for the effect of prostaglandins. The possibility of a rearrangement of the extracellular matrix is supported by the long duration of the effect of latanoprost after chronic treatment. The IOP will only slowly - over 2-3 weeks - return to pretreatment levels after 6 months' treatment with latanoprost, ${ }^{24}$ and this cannot be explained by any depot effect of the drug in the eye since it is not bound to melanin. However, other mechanisms are likely to be involved since the maximal effect of latanoprost and $\mathrm{PGF}_{2 \alpha}$ is reached within $8-12 \mathrm{~h}$, a time that is likely to be too short for a full effect mediated through induction of MMPs.

Considering the antagonistic effects on uveoscleral flow of cholinergic agonists and prostaglandin analogues one might expect that treatment with pilocarpine would block the effect of latanoprost. However, this is not the case. Even pre-treatment with strong doses of physostigmine failed to prevent the effect of latanoprost in the human eye. ${ }^{25}$ The explanation seems to be that an intensive contraction of the ciliary muscle is only seen for about $30 \mathrm{~min}$ after a single dose of a cholinergic agonist. Thus most of the time the ciliary muscle would be more or less relaxed and there is nothing to prevent latanoprost reaching the receptor and inducing the expected change in the ciliary muscle.

\section{Conclusions}

The recent introduction of prostaglandin analogues in the treatment of glaucoma has demonstrated that increasing uveoscleral flow is an unexpectedly effective way of reducing the IOP. We can expect an intensive search for new drugs, based on prostaglandin or other pharmacological principles, to find other drugs where this mechanism of action is used, focusing the interest of ophthalmologists on a new area: the extracellular matrix. 


\section{References}

1. Bill $\mathrm{A}$. The aqueous humor drainage mechanism in the cynomolgus monkey (Macaca irus) with evidence for unconventional routes. Invest Ophthalmol 1965;4:911-9.

2. Bill A, Hellsing K. Production and drainage of aqueous humor in the cynomolgus monkey (Macaca irus). Invest Ophthalmol 1965;4:920-6.

3. Bill A, Phillips CI. Uveoscleral drainage of aqueous humor in human eyes. Exp Eye Res 1971;12:275-81.

4. Brubaker RF. Flow of aqueous flow in humans. The Friedenwald lecture. Invest Ophthalmol Vis Sci 1991;32:3145-66.

5. Sperber GO, Bill A. A method for near-continuous determination of aqueous humor flow: effects of anaesthetics, temperature and indomethacin. Exp Eye Res 1984;39:435-53.

6. Toris CB, Camras CB, Yablonski ME. Effects of PhXA41, a new prostaglandin $F_{2 \alpha}$ analog, on aqueous humor dynamics in human eyes. Ophthalmology 1993;100:1297-304.

7. Poyer JF, Gabelt BT, Kaufman PL. The effect of topical PGF $_{2 \alpha}$ on uveoscleral outflow and outflow facility in the rabbit eye. Exp Eye Res 1992;54:277-83.

8. Toris CB, Yablonski ME, Wang Y-L, Camras CB. Aqueous humor of dynamics in the aging human eye.

Am J Ophthalmol 1999;127:407-12.

9. Bill A. Capillary permeability to and extravascular dynamics of myoglobin, albumin and gammaglobulin in the uvea. Acta Physiol Scand 1968;73:204-19.

10. Inomata $\mathrm{H}$, Bill $\mathrm{A}$. Exit sites of uveoscleral flow of aqueous humor in cynomolgus monkey eyes. Exp Eye Res 1977;25:113-8.

11. Bill A. Further studies on the influence of the intraocular pressure on aqueous humor dynamics in cynomolgus monkeys. Invest Ophthalmol 1967;6:364-72.

12. Törnquist P. Capillary permeability in cat choroid studied with the single injection technique. Acta Physiol Scand 1979;106:425-30.

13. Bill A, Wålinder PE. The effect of pilocarpine on the dynamics of aqueous humor in a primate (Macaca irus) Invest Ophthalmol 1966;5:170-5.
14. Bill A. Effects of atropine and pilocarpine on aqueous humor dynamics in cynomolgus monkey (Macaca irus). Exp Eye Res 1967;6:120-5.

15. Topper JE, Brubaker RF. Effects of timolol, epinephrine, and acetazolamide on aqueous flow during sleep. Invest Ophthalmol Vis Sci 1985;26:1315-9.

16. Bill A. Early effects of epinephrine on aqueous humor dynamics in vervet monkeys (Cercopithecus ethiops). Exp Eye Res 1969;8:35-43.

17. Camras CB, Feldman SG, Podos SM, Christensen RE Gardner SK, Fazio DT. Inhibition of the epinephrine-induced reduction of intraocular pressure by systemic indomethacin in humans. Am J Ophthalmol 1985;100:169-75.

18. Crawford K, Kaufman PL, Gabelt BT. Effects of topical PGF 20 on aqueous humour dynamics in cynomolgus monkeys. Curr Eye Res 1987;6:1035-44.

19. Stjernschantz J, Selén G, Sjöquist B, Resul B. Preclinical pharmacology of latanoprost, a phenylsubstituted $\mathrm{PGF}_{2 a}$ analogue. In: Samuelsson B, editor. Advances in prostaglandin, thromboxane, and leukotriene research, vol 23. New York: Raven Press, 1995:513-8.

20. Poyer JF, Millar C, Kaufman PL. Prostaglandin $F_{2 \alpha}$ effects on isolated rhesus monkey ciliary muscle. Invest Ophthalmol Vis Sci 1995;36:2461-5.

21. Lindsey JD, Kashiwagi K, Boyle D, Kashiwagi F, Firestrin F, Weinreb RN. Prostaglandins increase proMMP-1 and proMMP-3 secretion by human ciliary smooth muscle cells. Curr Eye Res 1996;15:869-75.

22. Ocklind A. Effect of latanoprost on the extracellular matrix of the ciliary muscle: a study on cultured cells and tissue sections. Exp Eye Res 1998;67:179-91.

23. Sagara T, Gaton DD, Lindsey JD, Gabelt BT, Kaufman PL, Weinreb $\mathrm{RN}$. Topical prostaglandin $\mathrm{F}_{2 \alpha}$ treatment reduces collagen types I, III, and IV in the monkey uveoscleral outflow pathway. Arch Ophthalmol 1999;117:794-801.

24. Lindén C, Nuija E, Alm A. Effects of intraocular pressure restoration and blood aqueous barrier after long-term treatment with latanoprost in POAG and ocular hypertension. Br J Ophthalmol 1997;81:370-2.

25. Lindén C, Alm A. Latanoprost and physostigmine have mostly additive ocular hypotensive effects in human eyes. Arch Ophthalmol 1997;115:857-61. 